\title{
Increased polyphenols and antioxidant activity of rice bean (Vigna umbellata L.) sprouts induced by Methyl Jasmonate: the promotion effect of Methyl Jasmonate on rice bean sprouts
}

\author{
Li LI' ${ }^{1}$, Xiaoyan GONG ${ }^{1}$, Hankun REN ${ }^{1,2}$, Xiaoyue WANG ${ }^{1}$, Yifan $\mathrm{HE}^{1}$, Yinmao DONG ${ }^{1 \star}$
}

\begin{abstract}
Rice bean (Vigna umbellata L.) sprouts are richer in metabolites and biological activities after germination. The total polyphenol and total flavonoid contents were analyzed during the sprouting process. The antioxidant activities of the sprouts were tested using 1,1-diphenyl-2- picrylhydrazyl radical (DPPH) screening assay. To determine the reason for increased activity, quantitative analysis of 8 flavonoids, in the germination process of methyl jasmonate (MeJA)-treated and control groups, was performed using high-performance liquid chromatography coupled with diode array detection (HPLC/DAD) and partial least squares discriminant analysis (PLS/DA). The metabolites in MeJA-treated beans shows that addition of $100.0 \mu \mathrm{mol}$ MeJA significantly increased the total flavonoid and polyphenol contents during the sprouting process. DPPH screening assay and analytical results show that MeJA has a significant influence on the production of secondary metabolites in sprouts between the $4^{\text {th }}$ and $6^{\text {th }}$ days, thereby inducing increased antioxidant activity. Flavonoids rutin, kaempferol-3-O-rutinoside, daidzin, and genistin are further identified as the main contributors to the increased DPPH screening activity.
\end{abstract}

Keyword: rice bean; Methyl Jasmonate; germination; polyphenols; antioxidant.

Practical Application: The influences of MeJA on the activities and total polyphenol content. of rice bean sprouts.

\section{Introduction}

Rice bean (Vigna umbellata L.), also known as the climbing mountain bean, mambi bean, and oriental bean, is native to Southeast Asia. It is consumed as a vegetable or as a pulse mixed with rice (Saikia et al., 1999). Legumes attract much research interest as a functional food ingredient because the proteins, saponins, and phenolic compounds found in them are active ingredients responsible for numerous health benefits (Yao et al., 2011).

Phenolic acids and flavonoids are major classes of metabolites found in rice bean. Three phenolic acids ( $p$-coumaric acid, ferulic acid, and sinapic acid) and five flavonoids (catechin, epicatechin, vitexin, isovitexin, and quercetin) have been identified in different rice bean samples. The dominant phenolic compound in all varieties was found to be vitexin, followed by catechin and isovitexin (Yao et al., 2012). Phenolic compounds have been reported to reduce the risks of cancer, heart disease, and diabetes, and shown to possess antibacterial, antiviral, anti-inflammatory, properties (Sutivisedsak et al., 2010).

Seed germination begins with water absorption. Significant changes occur during germination, including the interconversion of compounds and the synthesis of new compounds (Cevallos-Casals \& Cisneros-Zevallos, 2010). The plant hormone jasmonate and its methyl ester-methyl jasmonate (MeJA) - play important roles in the regulation of plant growth and in endogenous and exogenous stress signaling. Application of exogenous MeJA has increased the abundance of proteins associated with energy metabolism, which confers a protective role against stress to the plant and continues to provide energy for maintaining normal physiological processes (Farooq et al., 2018). The effects of MeJA on the production of secondary metabolites (alkaloids, polysaccharides, and flavonoids) have also been reported (Jiao et al., 2018).

To improve the active secondary metabolites of rice beans sprouts, the influence of MeJA on the metabolite profile and antioxidant activities was investigated during the sprouting process. The results wish to be beneficial to utilize rice bean sprouts as the raw materials for functional foods, health products, and cosmetics.

\section{Materials and methods}

\subsection{Chemicals and regents}

Rice beans (V. umbellate) were purchased from the local market (Beijing, China). DPPH and Folin-Ciocalteu reagent were obtained from Biodee Biotechnology Co., Ltd. (Beijing, China). MeJA and gallic acid (GAE) were purchased from Sigma Aldrich (St. Louis, MO, U.S.A.). HPLC-grade methanol was purchased from Merck (Germany). Ultra-pure water (18.2 M $\Omega$ ) was prepared with a Milli-Q water purification system (Millipore, Bedford, MA, USA). 
The flavonoid references were purchased from Shanghai TAUTO Biotech. Co., Ltd, all of which had $>98 \%$ purity by HPLC analysis. For HPLC/DAD quantitative analysis, the standards contained rutin $(75.83 \mu \mathrm{g} / \mathrm{mL})$, isoquercitrin $(90.00 \mu \mathrm{g} / \mathrm{mL})$, kaempferol-3-O-rutinoside $(86.66 \mu \mathrm{g} / \mathrm{mL})$, genistein $(74.16 \mu \mathrm{g} / \mathrm{mL})$, daidzin $(97.50 \mu \mathrm{g} / \mathrm{mL})$, glycitein $(63.33 \mu \mathrm{g} / \mathrm{mL})$, genistin $(80.00 \mu \mathrm{g} / \mathrm{mL})$, and daidzein $(80.00 \mu \mathrm{g} / \mathrm{mL})$ dissolved in $1.5 \mathrm{~mL}$ of $75 \%(v / v)$ methanol.

The solutions were stored in dark glass bottles at $4{ }^{\circ} \mathrm{C}$. The working standard solutions were freshly prepared by diluting suitable amounts of the above solutions with $75 \%(v / v)$ methanol before injection. The standard solutions and the sprout extracts were filtered through a $0.45-\mu \mathrm{m}$ filter prior to injection, and a $5.0-\mu \mathrm{L}$ volume of each was used for quantitative analysis.

\subsection{Cultivation of sprouts}

The rice bean seeds $(10.0 \mathrm{~g})$ were germinated using a previously reported method with some modifications (Tang et al., 2014). The germinating seeds were kept moist in an incubator without light at $26^{\circ} \mathrm{C}$. Sterile water or diluted MeJA $(30 \mathrm{~mL})$ was sprayed onto the sprouts every $24 \mathrm{~h}$ for 6 days. Sprout samples (1.0 g) from the MeJA-treated groups and control group were removed every $24 \mathrm{~h}$ for 6 days. There were 3 replicates of each treatment.

\subsection{Sample preparation for analysis}

The crushed 1.0-g samples were extracted by ultrasonication in $5.0 \mathrm{~mL}$ of $75 \%(v / v)$ methanol for $30 \mathrm{~min}$. The extracts were centrifuged at 12,000 rpm for $5 \mathrm{~min}$, and the supernatants of the samples were used for chemical and biological analysis. A small portion of the supernatant was passed through a $0.45-\mu \mathrm{m}$ filter, and a 5.0- $\mu \mathrm{L}$ volume was injected for HPLC/DAD quantitative analysis.

\subsection{Determination of total polyphenol content}

The total polyphenol contents in the MeJA-treated sprouts and the control group were measured over 6 days by Folin-Ciocalteu colorimetry (Singleton \& Rossi, 1965). The Folin-Ciocalteu reagent was prepared by diluting the commercial reagent concentrate in a 1:2 ratio with water. The supernatant of the sample $(0.1 \mathrm{~mL})$ was added to $0.1 \mathrm{~mL}$ methanol $/ 0.3 \% \mathrm{HCl}$ $(6: 4, v / v)$, and that solution was added to $10 \% \mathrm{Na}_{2} \mathrm{CO}_{3}(2.0 \mathrm{~mL})$. The Folin-Ciocalteu reagent $(0.1 \mathrm{~mL})$ was added to the mixture, which was then kept in the dark for $30 \mathrm{~min}$. The samples were shaken thoroughly, and the absorbance was measured at $750 \mathrm{~nm}$ using a microplate reader. The standard curve was constructed using gallic acid (mg GAE/g).

\subsection{Determination of total flavonoid content}

The total flavonoid contents in MeJA treatments and the control group were determined over 6 days using a previously reported colorimetric method (Chang et al., 2002). Briefly, $0.4 \mathrm{~mL}$ of $\mathrm{NaNO}_{2}$ $(5 \%, v / v)$ was added to $1.0 \mathrm{~mL}$ of the appropriately diluted samples. To this, $0.4 \mathrm{~mL}$ of $\mathrm{AlCl}_{3}(10 \%, v / v)$ was added after $6 \mathrm{~min}$, and $4.0 \mathrm{~mL}$ of $\mathrm{NaOH}(4 \%, v / v)$ and ethanol $(60 \%, v / v)$ were added to $10 \mathrm{~mL}$ of the resulting solution after $6 \mathrm{~min}$. The absorbance was measured at $508 \mathrm{~nm}$ using a microplate reader after $15 \mathrm{~min}$. A standard curve was constructed using rutin. The total flavonoid content was expressed as $\mathrm{mg}$ of rutin equivalent per $\mathrm{g}$ of the samples. The samples were analyzed in triplicate.

\subsection{DPPH radical scavenging activity}

Radical scavenging rates by the samples were measured by DPPH assay (Li et al., 2006). Crushed fragments of sprouts $(1.0 \mathrm{~g}$, removed at various times from 0 to $168 \mathrm{~h}$ ), along with $5.0 \mathrm{~mL}$ water, were ultrasonicated for $30 \mathrm{~min}$, and centrifuged at $5000 \mathrm{rpm}$ for $5.0 \mathrm{~min}$. For each sample, a $0.5-\mathrm{mL}$ aliquot of different concentrations was added to $1.0 \mathrm{~mL}$ DPPH solution $(100 \mu \mathrm{M})$ for $30 \mathrm{~min}$. Methanol was used as a blank solution. The decrease in absorbance was measured at $517 \mathrm{~nm}$. DPPH radical scavenging activity was expressed as the percentage (\%) of absorbance disappearance [(ADPPH - AS) /ADPPH] $\times 100$, where AS is the absorbance of the solution when the sample extract of a particular concentration has been added and ADPPH is the absorbance of the DPPH solution.

\subsection{Quantitation of the 8 flavonoids in the control and MeJA treated groups}

Chromatographic separation was performed using an Agilent 1260LC series system (Agilent Technologies, Palo Alto, CA, USA) equipped with an online vacuum degasser, quaternary pump, autosampler, thermostated column compartment, and a DAD. Agilent technologies Chemstation software for LC (B.02.01) was used. HPLC separation was performed on Agilent Eclipse Plus $\mathrm{C} 18$ column $(4.6 \mathrm{~mm} \times 250 \mathrm{~mm}, 5 \mu \mathrm{m})$. The detection wavelength was set at $254 \mathrm{~nm}$. The mobile phase consisted of water/acetic acid $(99.8: 0.2, v / v)(\mathrm{A})$ and acetonitrile (B). The gradient program consisted of $10-15 \%$ (B) for $0-20 \mathrm{~min}, 15-22 \%$ (B) for $20-30 \mathrm{~min}$, $22-25 \%$ (B) for $30-40 \mathrm{~min}, 25-30 \%$ (B) for $40-50 \mathrm{~min}, 30-40 \%$ (B) for $50-60 \mathrm{~min}$, and $40-100 \%$ (B) for 60-65 min. The flow rate was $1.0 \mathrm{~mL} / \mathrm{min}$ and the column temperature was set at $30^{\circ} \mathrm{C}$. Peak assignments were made for each single compound injection by comparing their retention times and characteristic absorption spectra obtained from the DAD with those of the authentic standards. The newly established HPLC/DAD method was used to simultaneously determine 8 flavonoids in sprouts at different points in time over 6 days. The flavonoid contents were calculated using the standard curves. Representative chromatograms of the samples are shown in Figure 1.

\subsection{Statistical analysis}

The data was analyzed by one-way analysis of variance (ANOVA) using SPSS17.0. Dunnett's t-test was used to analyze the significant difference among the groups, with significance level set as $p<0.05$.

\section{Results and discussion}

\subsection{Total polyphenols and flavonoids content in MeJA-treated sprouts}

Although no obvious differences were observed in the physical appearances of MeJA-treated and control group sprouts, MeJA treatment significantly affected polyphenol metabolism 
in the sprouts. The variations in total polyphenol and total flavonoid contents during the sprouting process are summarized in Table 1. The total flavonoid and total polyphenol contents were observed to increase significantly from the $2^{\text {nd }}$ day to the $6^{\text {th }}$ day upon treatment with $100.0 \mu \mathrm{mol}$ of MeJA; therefore, $100.0 \mu \mathrm{mol}$ was chosen as the optimal amount of MeJA. On the other hand, $1.0 \mathrm{mmol} \mathrm{MeJA}$ decreased the metabolite contents during the sprouting process. The use of $100.0 \mu \mathrm{mol}$ $\mathrm{MeJA}$ as the optimum intervention reagent for enhancing polyphenol content and activity has been frequently reported in literature (Lee et al., 2013). For example, $100.0 \mu \mathrm{mol} \mathrm{MeJA}$ was used to intervene in the sprouting process of Fagopyrum esculentum (buckwheat), resulting in a significant increase in total phenolic acid content.

\subsection{DPPH radical scavenging activity of MeJA-treated sprouts}

The results of DPPH radical scavenging assay (Table 2) show that $100.0 \mu \mathrm{mol}$ MeJA significantly increases the activities of sprouts between the $4^{\text {th }}$ and $6^{\text {th }}$ days. It is concluded that the increased polyphenol content from the $2^{\text {nd }}$ day enhanced antioxidant activity during the sprouting process. In a previous report, MeJA intervention was also shown to significantly increase the clearance rate of DPPH and ABTS free radicals, exhibited by a Lactuca sativa extract, indicating that intervention by an optimized concentration of MeJA increases the antioxidant activity of plant materials (Kim et al., 2007). The increased activity may also be related to the accumulation of flavonoids. We further quantified the change in flavonoid content and summarized the main contributors to the increased activities by HPLC/DAD with PLS/DA methods.

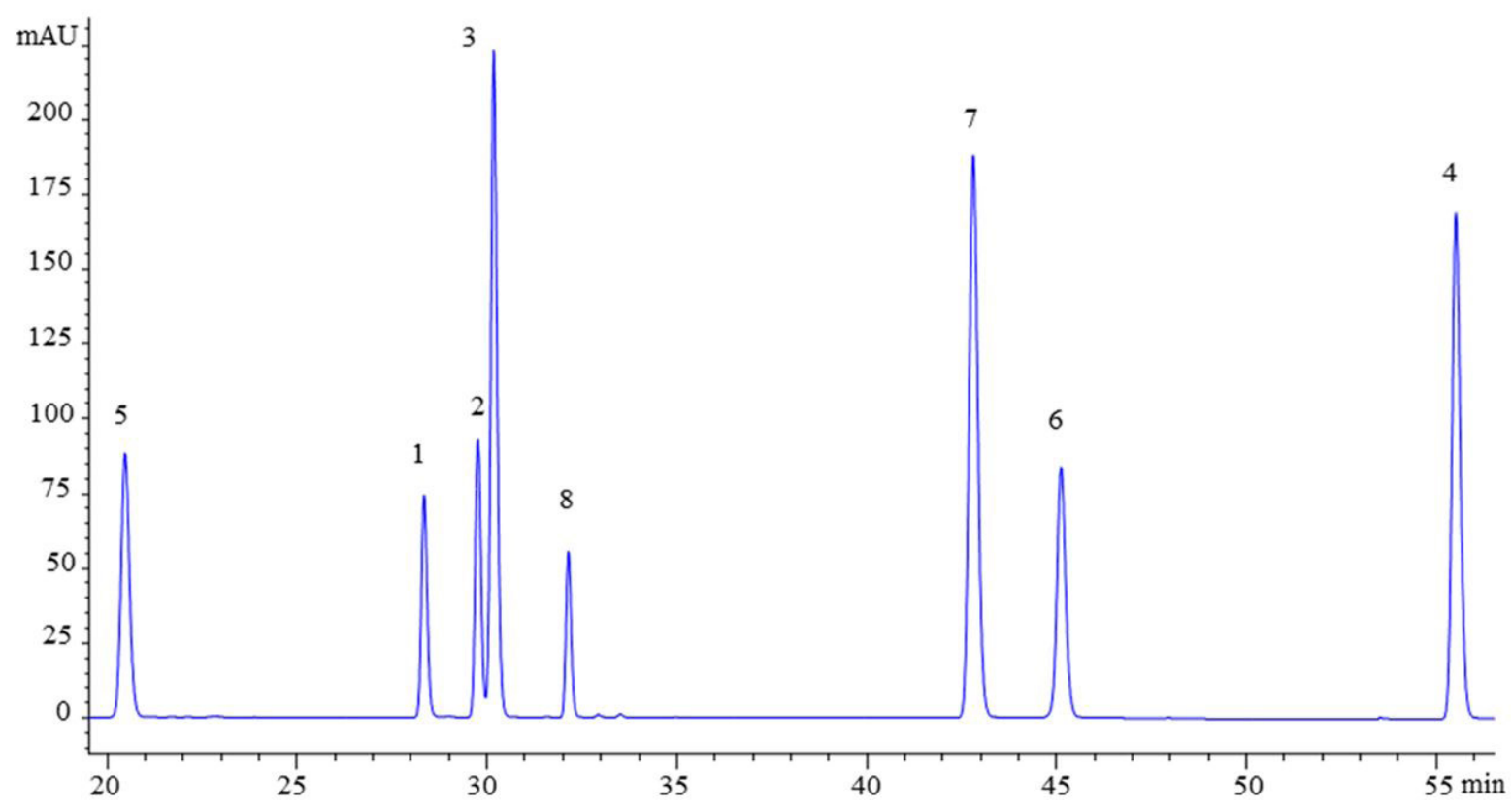

Figure 1. The chromatogram of mixed standard compounds: rutin (1), isoquercitrin (2), kaempferol-3-O-rutinoside (3), genistein (4), daidzin (5), glycitein (6), genistin (7), and daidzein (8).

Table 1. The total polyphenol and total flavonoid contents upon intervention by different concentrations of MeJA ( $\mathrm{n}=3$ ).

\begin{tabular}{|c|c|c|c|c|c|c|c|c|}
\hline \multirow{2}{*}{ MeJA concentration } & \multicolumn{4}{|c|}{ Total polyphenols $(\mathrm{mg} / \mathrm{g})$} & \multicolumn{4}{|c|}{ Total flavonoids (mg/g) } \\
\hline & Day 0 & Day 2 & Day 4 & Day 6 & Day 0 & Day 2 & Day 4 & Day 6 \\
\hline $0 \mu \mathrm{moL}$ & $1.96 \pm 0.06 \mathrm{a}$ & $2.43 \pm 0.14 \mathrm{a}$ & $4.18 \pm 0.05 a$ & $4.47 \pm 0.08 \mathrm{a}$ & $1.34 \pm 0.11 \mathrm{a}$ & $1.56 \pm 0.17 b$ & $1.92 \pm 0.22 \mathrm{a}$ & $2.06 \pm 0.32 b$ \\
\hline $100.0 \mu \mathrm{mol}$ & - & $2.75 \pm 0.11 \mathrm{a}$ & $4.35 \pm 0.28 b$ & $4.65 \pm 0.02 \mathrm{a}$ & - & $1.87 \pm 0.28 \mathrm{a}$ & $2.07 \pm 0.18 \mathrm{a}$ & $2.58 \pm 0.14 \mathrm{a}$ \\
\hline $1.0 \mathrm{mmol}$ & - & $1.86 \pm 0.05 b$ & $3.62 \pm 0.09 b$ & $4.27 \pm 0.05 a$ & - & $0.90 \pm 0.06 c$ & $0.99 \pm 0.13 b$ & $2.00 \pm 0.21 \mathrm{~b}$ \\
\hline
\end{tabular}

Values are a mean \pm SD $(n=3)$. Duncan's multiple range test was used to analyzed the differences of the groups. Different characters in the same line are $p<0.05$.

Table 2. The DPPH clearance rate (\%) of MeJA-treated group $(100.0 \mu \mathrm{mol})$ and the control groups.

\begin{tabular}{cccc}
\hline Groups & Day 2 & Day 4 & Day 6 \\
\hline Control & $43.44 \pm 2.29$ & $50.97 \pm 2.55$ & $51.29 \pm 2.56$ \\
MeJA-treated & $47.91 \pm 2.39$ & $79.68 \pm 4.82^{* *}$ & $65.89 \pm 3.60^{*}$ \\
\hline
\end{tabular}

Values are a mean \pm SD $(\mathrm{n}=3)$. Duncan's multiple range test was used to analyze the differences of the groups; ${ }^{*}$ indicated $p<0.05 ;{ }^{* *}$ indicated $p<0.01$. 


\subsection{Quantitation of the 8 flavonoids using HPLC/DAD}

\section{Quantitative method validation}

Acetonitrile/water and methanol/water in different ratios were investigated as the mobile phase. Aqueous acetic acid $(0.2 \%, v / v)$ and acetonitrile eluted all 8 standard compounds with baseline separation. Based on the absorption maxima of the standards in the UV spectra acquired by DAD, the monitoring wavelength was set at $254 \mathrm{~nm}$. The column temperature was kept constant at $30{ }^{\circ} \mathrm{C}$. The linearity, precision (intra-day and inter-day assays), stability, and recovery were evaluated according to guidelines of ICH Topic Q2B (summarized in Table 3). The limits of detection (LOD) and quantification (LOQ) were calculated as the concentrations of injected sample with signal-to-noise ratios of 3 and 10, respectively. The verified reversed-phase HPLC method was shown to be a precise, accurate, and reliable technique to quantitatively evaluate the main flavonoids in the sprouts. For quantitative analysis, the levels of 8 flavonoids $(\mu \mathrm{g} / \mathrm{g})$ in MeJA-treated and control samples over 6 days were measured, and the results are given in Table 4.

\section{Multivariate statistical analysis}

To compare the differences in metabolite profiles of the MeJA-treated and control groups, a PLS-DA plot was obtained using the SIMCA-P software package. The quantitative results for the 8 flavonoids were analyzed by the PLS-DA plot to visualize the differences among samples (Figure 2A).

It is indicated that the MeJA-treated sprouts can be clearly distinguished from the control group sprouts from the $2^{\text {nd }}$ day onwards. The MeJA-treated groups can be differentiated according to the quantitative results of the 8 flavonoids (Figure 2B). The flavonoids rutin, kaempferol-3-O-rutinoside, daidzin, and genistin are further identified as the main contributors to the increased DPPH screening activity. MeJA has a more significant influence on the production of secondary metabolites in sprouts from the $4^{\text {th }}$ to $6^{\text {th }}$ days, inducing the increased DPPH screening activity (Figure 2C).

\section{Flavonoids in MeJA-treated and the control samples}

During the sprouting process, the flavonoids changed due to enzyme activity. The flavonoids rutin, isoquercitrin, and kaempferol-3-O-rutinoside are only detected in the $1^{\text {st }}$ day sprout samples of the control groups. MeJA induces the production of the flavonoids genistein, daidzin, and daidzein from the $1^{\text {st }}$ day.

The rutin content of the control group shows no observable change during the sprouting process. In the MeJA-treated groups, rutin is not detected in the first two days, but rutin content increases rapidly from the $3^{\text {rd }}$ day to the $6^{\text {th }}$ day, and is significantly higher than that of the control groups on the $5^{\text {th }}$ and $6^{\text {th }}$ day, indicating that MeJA could promote rutin synthesis. Rutin has many biological

Table 3. Results of regression analysis on calibration curves, detection limits, precision and repeatability.

\begin{tabular}{|c|c|c|c|c|c|c|c|}
\hline \multirow{2}{*}{ NO. } & \multirow{2}{*}{$\begin{array}{c}\text { Calibration } \\
\text { curves }\end{array}$} & \multirow{2}{*}{$\mathrm{R}^{2}$} & Linear range & LOD & LOQ & Precision & Repeatability \\
\hline & & & $(\mu \mathrm{g} / \mathrm{g})$ & $(\mu \mathrm{g} / \mathrm{g})$ & $(\mu \mathrm{g} / \mathrm{g})$ & $(\mathrm{RSD}, \%)$ & $(\mathrm{RSD}, \%)$ \\
\hline rutin & $y=8.950 x-5.425$ & 0.999 & $5.62-90.00$ & 0.32 & 1.06 & 0.82 & 4.15 \\
\hline isoquercitrin & $y=11.46 x-7.1$ & 0.999 & $5.41-86.67$ & 0.16 & 0.52 & 0.89 & 2.97 \\
\hline kaempferol-3-O-rutinoside & $y=34.75 x-9.25$ & 0.999 & $4.63-71.16$ & 0.24 & 0.71 & 0.89 & 2.31 \\
\hline genistein & $y=32.23 x-7.795$ & 0.999 & $5.00-80.00$ & 0.25 & 0.82 & 0.17 & 1.05 \\
\hline daidzin & $y=18.18 x-4.654$ & 0.999 & $4.74-75.83$ & 0.26 & 0.86 & 0.96 & 2.97 \\
\hline glycitein & $y=17.91 x-79.61$ & 0.999 & $5.00-80.00$ & 0.099 & 0.33 & 1.13 & 1.05 \\
\hline genistin & $y=5.514 x-2.308$ & 0.999 & $6.09-97.50$ & 0.081 & 0.27 & 0.12 & 1.33 \\
\hline daidzein & $y=47.37 x-10.69$ & 0.999 & $3.96-63.33$ & 0.225 & 0.75 & 0.3 & 1.33 \\
\hline
\end{tabular}

Table 4. The contents the 8 flavonoids in MeJA-treated and the control groups from day1 to day 6 ( $\mu \mathrm{g} / \mathrm{g}$ ).

\begin{tabular}{|c|c|c|c|c|c|c|c|c|}
\hline Samples & rutin & isoquercitrin & $\begin{array}{l}\text { kaempferol-3- } \\
\text { O-rutinoside }\end{array}$ & genistein & daidzin & glycitein & genistin & daidzein \\
\hline MeJA-1 & - & 16.87 & 31.39 & 8.02 & 62.65 & - & - & 3.63 \\
\hline MeJA-2 & - & 11.88 & 18.97 & 5.25 & 34.45 & - & - & 2.77 \\
\hline MeJA-3 & 17.3 & 16.19 & 23.05 & 7.06 & 59.37 & - & 30.24 & 3.14 \\
\hline MeJA-4 & 22.68 & 13.77 & 22.11 & 9.77 & 31.56 & 3.76 & 32.9 & 3.81 \\
\hline MeJA-5 & 39.48 & 12.35 & 17.47 & 10.87 & 56.84 & 5.23 & 31.95 & 5.48 \\
\hline MeJA-6 & 50.44 & 12.64 & 17.69 & 11.31 & 164.33 & 6.32 & 43.27 & 6.83 \\
\hline Control-1 & 18.30 & 18.28 & 4.50 & - & - & - & - & - \\
\hline Control-2 & 16.33 & 18.27 & 7.04 & 3.58 & - & - & - & - \\
\hline Control-3 & 16.23 & 25.36 & 7.87 & 12.32 & 10.94 & 35.97 & - & - \\
\hline Control-4 & 18.30 & 29.42 & 10.08 & 5.99 & 29.85 & 33.05 & 12.91 & 3.61 \\
\hline Control-5 & 15.79 & 32.52 & 12.22 & 7.63 & 51.57 & 29.60 & 13.00 & 5.81 \\
\hline Control-6 & 14.11 & 37.00 & 11.12 & 13.92 & 51.72 & 33.75 & 18.17 & 35.43 \\
\hline
\end{tabular}

“-” Indicated it wasn't detected. 
A

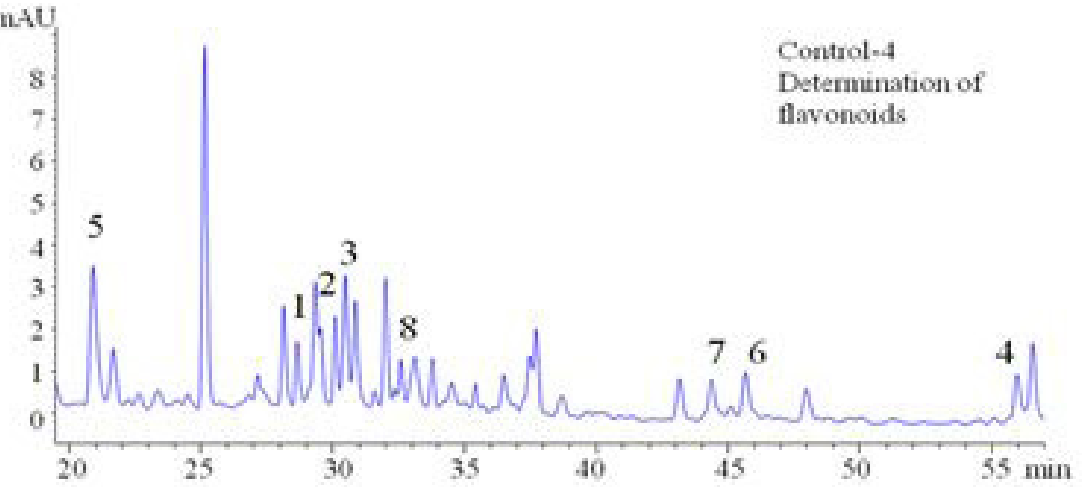

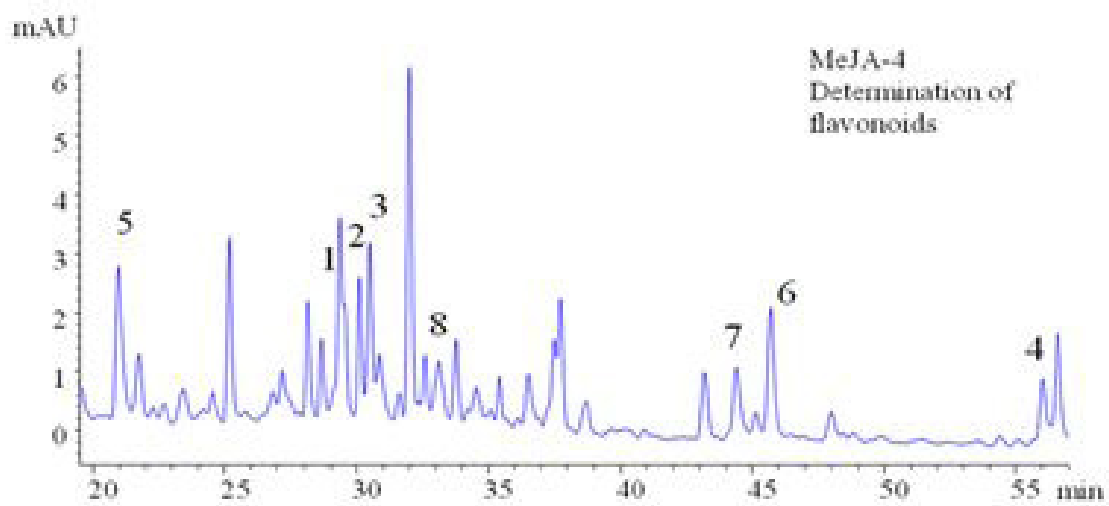

B

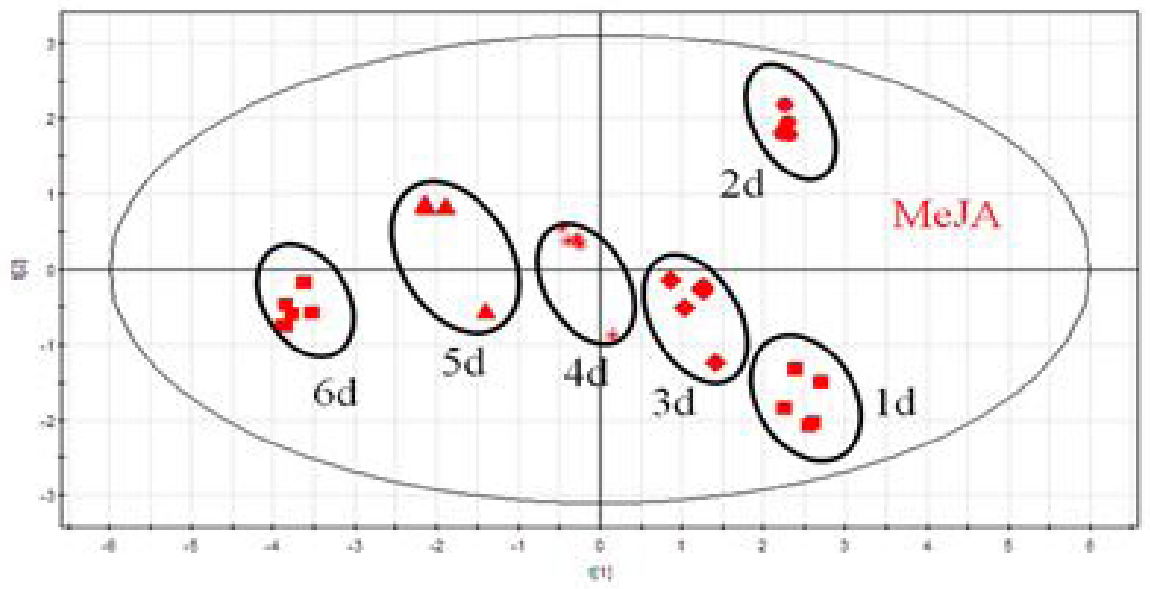

C

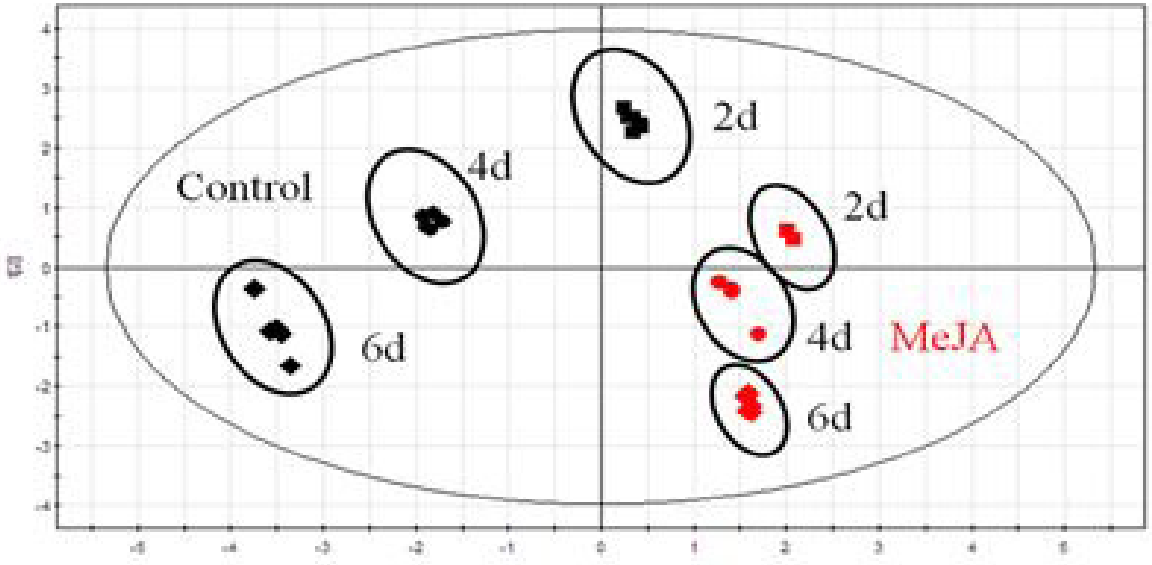

Figure 2. (A) Chromatograms of the $4^{\text {th }}$ day samples of the control and MeJA-treated groups; (B) PLS-DA score plots of MeJA-treated groups from day 1 to 6; (C) PLS-DA score plots of days 2, 4, and 6 of the control and MeJA-treated groups. 
activities, such as anti-oxidant properties, regulation of blood lipids, and weight reduction (Hsu \& Yen, 2006). Additionally, it is one of the probable contributors to increased DPPH screening activity in this study. With increasing rutin content of the extracts, the isoquercitrin content decreases significantly from the $2^{\text {nd }}$ day to the $6^{\text {th }}$ day. It is suggested that glycosidase enzymes, such as flavonol 3-O-glucosyl transferase (FGT), might be activated by MeJA intervention, leading to isoquercitrin combining with sugar units rhamnose (Rha) to convert into rutin (Petpiroon et al, 2015). The kaempferol-3-O-rutinoside content increases significantly from the $1^{\text {st }}$ day to the $6^{\text {th }}$ day. This also suggests that FGT is activated by MeJA intervention, promoting the transformation of the intermediate kaempferol to kaempferol-3-O-rutinoside. Kaempferol-3-O-rutinoside has a positive effect on the migration of human keratinocyte cells, which is a critical process in wound healing. The enhancement of cell motility by kaempferol-3-O-rutinoside is greater than that induced by its aglycone analogue, indicating the important role played by the rutinoside sugar moiety in the structure of the compound. It is also one of the probable contributors to the increased DPPH screening activity in this study. In addition, the contents of the isoflavones daidzin, glycitein, genistin, and daidzein show significant changes induced by MeJA intervention. Daidzin and genistin contents increase more significantly during the sprouting process. On one hand, the enzymes isoflavone synthase
(IFS) and isoflavone dehydratase (IFD) might be activated by MeJA treatment, promoting the transformation of intermediate flavonones into isoflavones instead of flavones. On the other hand, more metabolite sugars are produced by MeJA intervention, promoting the transformation of aglucons into the isoflavonoids daidzin and genistin. These soybean isoflavones have extensive biological activities, such as antioxidant properties, anticancer properties, blood lipid regulation, and estrogenic effects, which are beneficial to human health. Spraying MeJA onto the leaves of growing soybeans in the field has been reported to increase their total isoflavone content to $960 \mu \mathrm{g} / \mathrm{g}$ (Ma \& Zhao, 2011). This influence of MeJA might be related to some enzymes and genes, such as phenylalanine ammonia lyase, in the phenylalanine metabolic pathway (Andi et al., 2001). The potential influence of MeJA on the transformation pathway of the main polyphenols during the sprouting process is summarized in Figure 3.

\section{Conclusions}

Overall, intervention by MeJA enhances flavonoid metabolism and antioxidant activities in the rice bean sprouting process. Thus, MeJA is an excellent antioxidant synergist whose application provides a promising technique to produce foods and medicines with improved therapeutic properties.

\section{Shikimic acid}

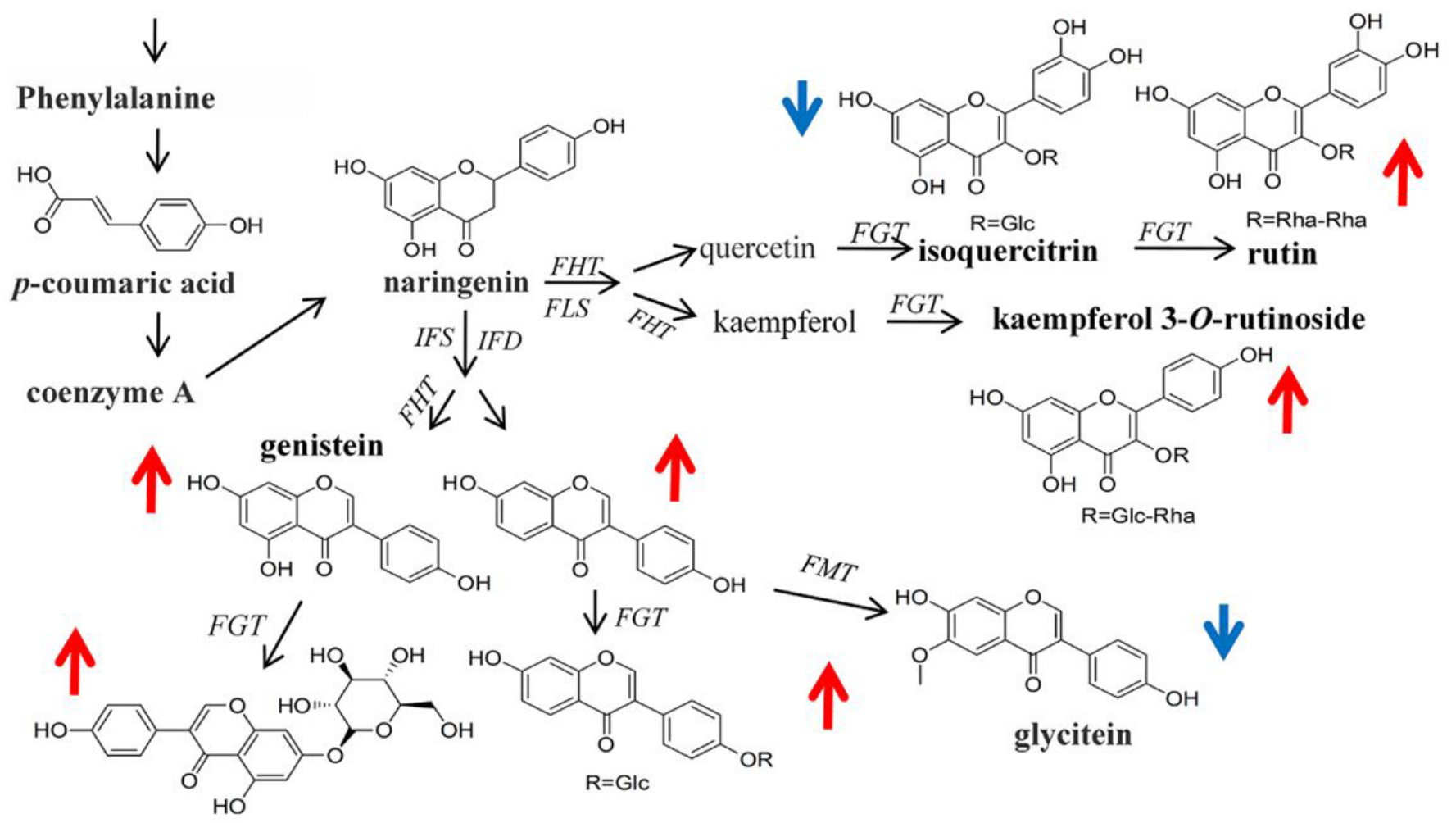

\section{genistin}

Figure 3. Influence of MeJA on schematic diagram of phenylpropanoid pathways (red arrows represent increasement; blue arrows represent decreasement; FHT = flavanone 3-hydroxylase; FGT = flavonol 3-O-glucosyl transferase; FLS = flavonol synthase; IFS = isoflavone synthase; IFD: isoflavone dehydratase; FMT = flavanone methylase). 


\section{Acknowledgements}

This work was supported by National Natural Science Foundations of China (31501402). This work was also supported by the Key Laboratory of Cosmetic, China National Light Industry, Beijing Technology and Business University Innovation Fund ID: KLC-2017-YB3.

\section{References}

Andi, S., Taguchi, F., Toyoda, K., Shiraishi, T., \& Ichinose, Y. (2001). Effect of methyl jasmonate on harpin-induced hypersensitive cell death, generation of hydrogen peroxide and expression of $P A L$ mRNA in tobacco suspension cultured by-2 cells. Plant \& Cell Physiology, 42(4), 446-449. http://dx.doi.org/10.1093/pcp/pce056. PMid:11333317.

Cevallos-Casals, B. A., \& Cisneros-Zevallos, L. (2010). Impact of germination on phenolic content and antioxidant activity of 13 edible seed species. Food Chemistry, 119(4), 1485-1490. http:// dx.doi.org/10.1016/j.foodchem.2009.09.030.

Chang, C. C., Yang, M. H., Wen, H. M., \& Chern, J. C. (2002). Estimation of total flavonoid content in propolis by two complementary colorimetric methods. Journal of Food and Drug Analysis, 10(3), 178-182.

Farooq, M. A., Zhang, K., Islam, F., Wang, J., Athar, H. U. R., Nawaz, A., Zafar, Z. U., Xu, J., \& Zhou, W. (2018). Physiological and iTRAQ based quantitative proteomics analysis of methyl jasmonate induced tolerance in Brassica napus under arsenic stress. Proteomics, 18(10), e1700290. http://dx.doi.org/10.1002/pmic.201700290. PMid:29528557.

Hsu, C. L., \& Yen, G. C. (2006). Induction of cell apoptosis in 3t3-11 pre-adipocytes by flavonoids is associated with their antioxidant activity. Molecular Nutrition \& Food Research, 50(11), 1072-1079. http://dx.doi.org/10.1002/mnfr.200600040. PMid:17039455.

Jiao, C., Song, C., Zheng, S., Zhu, Y., Jin, Q., Cai, Y., \& Lin, Y. (2018). Metabolic profiling of Dendrobium officinale in response to precursors and Methyl Jasmonate. International Journal of Molecular Sciences, 19(3), 728-747. http://dx.doi.org/10.3390/ijms19030728. PMid:29510516.

Kim, H. J., Fonseca, J. M., Choi, J. H., \& Kubota, C. (2007). Effect of methyl jasmonate on phenolic compounds and carotenoids of romaine lettuce (Lactuca sativa 1.). Journal of Agricultural and Food Chemistry, 55(25), 10366-10372. http://dx.doi.org/10.1021/ jf071927m. PMid:17990849.
Lee, Y. J., Kim, K. J., Park, K. J., Yoon, B. R., Lim, J. H., \& Lee, O. H. (2013). Buckwheat (Fagopyrum esculentum m.) sprout treated with methyl jasmonate (MeJA) improved anti-adipogenic activity associated with the oxidative stress system in 3t3-11 adipocytes. International Journal of Molecular Sciences, 14(1), 1428-1442. http:// dx.doi.org/10.3390/ijms14011428. PMid:23344050.

Li, Y., Song, Q., Chen, P., Zhao, W., \& Wang, S. (2006). Synthesis of hydroxystilbene derivatives and their antioxidant activity by DPPH method. Journal of Chemical Research in Chinese Universities, 22(6), 742-746. http://dx.doi.org/10.1016/S1005-9040(06)60202-X.

Ma, J. L., \& Zhao, Y. (2011). Effect of exogenous methyl jasmonate on pathway of isoflavone synthesis in soybean. The Journal of Northeast Agricultural University, 42(5), 14-18.

Petpiroon, N., Suktap, C., Pongsamart, S., Chanvorachote, P., \& Sukrong, S. (2015). Kaempferol-3-O-rutinoside from afgekia mahidoliae promotes keratinocyte migration through fak and rac1 activation. Journal of Natural Medicines, 69(3), 340-348. http://dx.doi.org/10.1007/ s11418-015-0899-3. PMid:25783411.

Saikia, P., Sarkar, C. R., \& Borua, I. (1999). Chemical composition, antinutritional factors and effect of cooking on nutritional quality of rice bean [Vigna umbellata (Thunb; Ohwi and Ohashi)]. Food Chemistry, 67(4), 347-352. http://dx.doi.org/10.1016/S03088146(98)00206-4.

Singleton, V. L., \& Rossi, J. A. (1965). Colorimetry of total phenolics with phosphomolybdic-phosphotungstic acid reagents. American Journal of Enology and Viticulture, 16(3), 144-158.

Sutivisedsak, N., Cheng, H. N., Willett, J. L., Lesch, W. C., Tangsrud, R. R., \& Biswas, A. (2010). Microwave-assisted extraction of phenolics from bean (Phaseolus vulgaris 1.). Food Research International, 43(2), 516-519. http://dx.doi.org/10.1016/j.foodres.2009.09.014.

Tang, D., Dong, Y., Guo, N., Li, L., \& Ren, H. (2014). Metabolomic analysis of the polyphenols in germinating mung beans (Vigna radiata) seeds and sprouts. Journal of the Science of Food and Agriculture, 94(8), 1639-1647. http://dx.doi.org/10.1002/jsfa.6471. PMid:24203396.

Yao, Y., Cheng, X. Z., Wang, L. X., Wang, S. H., \& Ren, G. (2012). Major phenolic compounds, antioxidant capacity and antidiabetic potential of rice bean (Vigna umbellata L.) in China. International Journal of Molecular Sciences, 13(3), 2707-2716. http://dx.doi.org/10.3390/ ijms13032707. PMid:22489119.

Yao, Y., Cheng, X. Z., Wang, L. X., Wang, S. H., \& Ren, G. X. (2011). Biological potential of sixteen legumes in China. International Journal of Molecular Sciences, 12(10), 7048-7058. http://dx.doi.org/10.3390/ ijms12107048. PMid:22072935. 